\title{
Persistent template effect in InAs/GaAs quantum dot bilayers
}

\author{
E. Clarke, ${ }^{1, a)}$ P. Howe ${ }^{1}$ M. Taylor, ${ }^{1}$ P. Spencer, ${ }^{1}$ E. Harbord,${ }^{1}$ R. Murray, ${ }^{1}$ \\ S. Kadkhodazadeh, ${ }^{2}$ D. W. McComb, ${ }^{2}$ B. J. Stevens, ${ }^{3}$ and R. A. Hogg $^{3}$ \\ ${ }^{1}$ Department of Physics, Blackett Laboratory, Imperial College London, Prince Consort Road, \\ London SW7 2AZ, United Kingdom \\ ${ }^{2}$ Department of Materials, Imperial College London, Exhibition Road, London SW7 2AZ, United Kingdom \\ ${ }^{3}$ School of Electronic and Electrical Engineering, University of Sheffield, Sheffield S1 3JD, \\ United Kingdom
}

(Received 2 November 2009; accepted 21 April 2010; published online 1 June 2010)

\begin{abstract}
The dependence of the optical properties of InAs/GaAs quantum dot (QD) bilayers on seed layer growth temperature and second layer InAs coverage is investigated. As the seed layer growth temperature is increased, a low density of large QDs is obtained. This results in a concomitant increase in dot size in the second layer, which extends their emission wavelength, reaching a saturation value of around $1400 \mathrm{~nm}$ at room temperature for GaAs-capped bilayers. Capping the second dot layer with InGaAs results in a further extension of the emission wavelength, to $1515 \mathrm{~nm}$ at room temperature with a narrow linewidth of $22 \mathrm{meV}$. Addition of more InAs to high density bilayers does not result in a significant extension of emission wavelength as most additional material migrates to coalesced InAs islands but, in contrast to single layers, a substantial population of regular QDs remains. ( 2010 American Institute of Physics. [doi:10.1063/1.3429226]
\end{abstract}

\section{INTRODUCTION}

There has been much interest in the growth of closely stacked quantum dot (QD) layers, for example, to optimize the design of device structures to maximize optical gain, ${ }^{1,2}$ to extend their emission wavelength, ${ }^{3,4}$ and also to develop novel applications, such as potential qubits for quantum information processing. ${ }^{5,6}$ When there is a sufficiently small separation between two QD layers, strain from the underlying layer influences the growth conditions of an upper layer, leading to preferential nucleation of QDs above buried QDs. ${ }^{7}$ This provides an additional control mechanism over the growth process and allows greater freedom of choice of QD properties in stacked QD layers than is possible for single layers. Typical growth parameters such as coverage, ${ }^{3}$ temperature, ${ }^{4}$ and growth rate $^{8}$ can be varied to tune the size and composition of QDs in the upper layer while keeping the QD density constant, since the lower (seed) layer acts as a template for QD nucleation. These InAs/GaAs QD bilayers have been successfully incorporated into edge-emitting laser structures with room temperature operation at wavelengths up to $1430 \mathrm{~nm}^{8}{ }^{8}$

In this paper we examine two aspects of the growth of InAs/GaAs QD bilayers. In the first part of the paper we investigate the effect of the seed layer growth conditions on the structural and optical properties of QD bilayers, with the aim of extending the QD emission wavelength. Techniques for growth of InAs/GaAs QDs with room temperature emission at $1300 \mathrm{~nm}$ are now well established, ${ }^{9-11}$ but further extension of InAs/GaAs QD emission wavelength toward the $1550 \mathrm{~nm}$ telecoms window is rarely reported. ${ }^{12-16}$ By variation in the growth temperature of the seed layer, the number density of seed QDs can be varied over a range of $40-300 \mu \mathrm{m}^{-2}$ in a simple and reproducible manner. This in

${ }^{a)}$ Electronic mail: edmund.clarke@imperial.ac.uk. turn results in a comparable change in QD density in the second layer of bilayer structures and an accompanying variation in the height of QDs. The larger QD size allows extension of the room temperature emission of the InAs/ GaAs QD bilayers from $1340 \mathrm{~nm}$ to around $1400 \mathrm{~nm}$ using GaAs capping. This range can be extended beyond $1500 \mathrm{~nm}$ when the second layer is InGaAs capped.

In the second part, we investigate increasing the InAs coverage in the second layer of high density QD bilayers. Since the extension of the emission wavelength of the QD bilayers we report in the first part of the paper is achieved by increasing the size of the second layer QDs, it is natural to assume that increasing the InAs coverage in the second layer of the high density bilayers may also result in a similar extension of their emission wavelength. However, previous studies of single QD layers show there is an optimum InAs coverage beyond which saturation in the emission wavelength occurs ${ }^{17}$ and, as the coverage is increased, large irregular islands form, in addition to the stable ensemble of coherent islands. ${ }^{18-20}$ Increasing the InAs coverage in the second layer of the bilayers does not result in a significant extension of the emission wavelength as the majority of the additional material accumulates in the large islands and the size of the regular, emissive QDs remains approximately constant. However, even at very high InAs coverage (9 monolayers (ML)), where the structure is dominated by apparently coalesced InAs islands, regular-sized InAs islands remain and there is surprisingly little degradation in the room temperature photoluminescence (PL) intensity obtained from these samples.

\section{EXPERIMENTAL DETAILS}

Samples were grown by molecular beam epitaxy on undoped $\mathrm{GaAs}(001)$ substrates. Following oxide removal and growth of a $200 \mathrm{~nm} \mathrm{GaAs}$ buffer, the GaAs surface was 
annealed at a substrate temperature of $580{ }^{\circ} \mathrm{C}$ under an $\mathrm{As}_{2}$ flux for $10 \mathrm{~min}$. The temperature was then reduced for growth of the seed QD layer by deposition of 2.4 ML InAs. An InAs growth rate of $0.014 \mathrm{ML} \mathrm{s}^{-1}$ was used for all the samples. The first study investigates the dependence of dot density on growth temperature of the seed layer in the range 475 to $515{ }^{\circ} \mathrm{C}$ (the lower and upper ends of this range are dictated by the quality of the GaAs capping layer and In desorption, respectively). Uncapped seed layer samples were used for atomic force microscopy (AFM) characterization, while completed structures were grown using the following recipe: the seed layer was capped by a $10 \mathrm{~nm}$ GaAs spacer layer grown at the same temperature and the sample was again annealed for $10 \mathrm{~min}$ at $580{ }^{\circ} \mathrm{C}$ in order to smooth the GaAs surface and desorb any segregated In. ${ }^{21}$ The temperature was then reduced to $467{ }^{\circ} \mathrm{C}$ for growth of $3.3 \mathrm{ML}$ InAs to form the second QD layer; this eliminates $\mathrm{In} / \mathrm{Ga}$ intermixing. ${ }^{4}$ For the second study the seed layer was grown at a temperature of $475{ }^{\circ} \mathrm{C}$ while the amount of InAs used to form the second layer covered the range 3.7-9.0 ML.

Samples for AFM characterization of the second layer were left uncapped but samples for PL were capped, either by $100 \mathrm{~nm} \mathrm{GaAs}$ or by a $4 \mathrm{~nm} \operatorname{In}_{0.26} \mathrm{Ga}_{0.74}$ As layer followed by the remaining $(96 \mathrm{~nm}$ ) GaAs cap. The first $15 \mathrm{~nm}$ of the capping layers was grown at $467{ }^{\circ} \mathrm{C}$ before the temperature was increased to the optimum GaAs growth temperature of $580{ }^{\circ} \mathrm{C}$. AFM measurements were performed using a Digital Instruments atomic force microscope in contact mode for the initial seed layer growth temperature study and tapping mode for the second layer InAs coverage study. Samples for transmission electron microscopy (TEM) characterization were prepared by mechanical polishing followed by Ar ion milling and images were obtained using JEOL 2010 TEM operating at $200 \mathrm{kV}$. PL spectra were obtained using a $\mathrm{HeNe}$ laser, dispersing the emitted light with a SPEX $0.5 \mathrm{~m}$ monochromator, with detection by a cooled Ge diode using standard lock-in techniques.

\section{RESULTS AND DISCUSSION}

\section{A. Effect of seed layer growth temperature}

The AFM images shown in Fig. 1 illustrate the variation in the size and density of QDs that can be obtained by changing the growth temperature from 480 to $515^{\circ} \mathrm{C}$. At $480{ }^{\circ} \mathrm{C}$, a relatively high density of $3.1 \times 10^{10} \mathrm{~cm}^{-2}$ of small islands (with an average island height of $6.1 \mathrm{~nm}$ and base diameter of $57 \mathrm{~nm}$ ) is formed, despite the low InAs growth rate used. At $515{ }^{\circ} \mathrm{C}$, a sixfold lower island density is obtained, with a corresponding increase in the size of the InAs islands to an average height of $15.1 \mathrm{~nm}$ and base diameter of $100 \mathrm{~nm}$. This is not surprising since the same amount of deposited In is now distributed among fewer islands and the higher growth temperature is likely to lead to increased $\mathrm{Ga}$ inclusion from the substrate. ${ }^{22} \mathrm{~A}$ similar change in the seed layer density can also be achieved by varying the InAs growth rate ${ }^{23}$ however, we have chosen to vary the growth temperature in order to maintain a constant In cell temperature (and InAs growth rate) for growth of both QD layers.
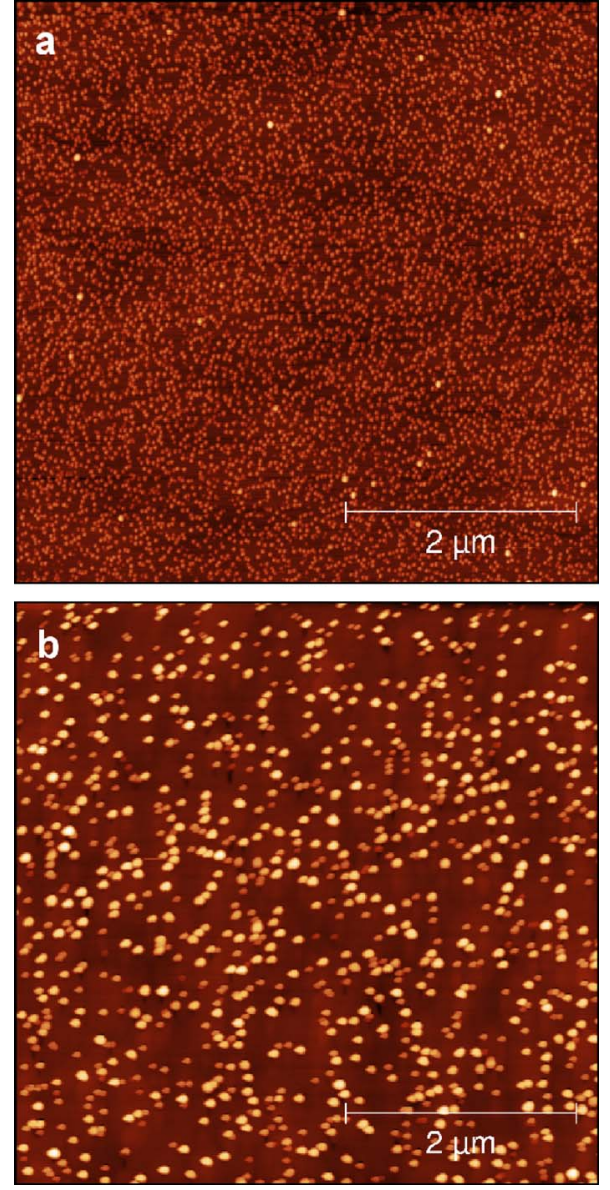

FIG. 1. (Color online) $5 \times 5 \mu \mathrm{m}^{2} \mathrm{AFM}$ images obtained from uncapped InAs/GaAs QD seed layers grown at (a) $480{ }^{\circ} \mathrm{C}$ and (b) $515^{\circ} \mathrm{C}$.

Figure 2(a) summarizes an analysis of AFM images obtained from uncapped seed layers and bilayers. It shows a monotonic reduction in island density in both layers as the seed layer growth temperature is increased. Both layers follow the same trend, with approximately equal densities in both layers over the whole temperature range, indicating that the seed layer acts as a highly effective template for the growth of the second layer QDs and that the majority of QDs in the bilayers must be vertically correlated. ${ }^{7}$ As the island density decreases there is a concomitant increase in the average height of islands in both layers, as shown in Fig. 2(b). It is well known that when islands are capped, they rapidly collapse in height but larger islands are expected to better maintain their height and have a larger In-content once capped. ${ }^{24}$ The inset to Fig. 3 shows a bright field TEM image obtained from a bilayer sample with a seed layer grown at $492{ }^{\circ} \mathrm{C}$. There is a clear vertical correlation between the QDs in the bilayer, and we can estimate a height of $\sim 5 \mathrm{~nm}$ for the seed layer QDs and 7-8 $\mathrm{nm}$ for QDs in the second layer. These are likely to be overestimates since contrast in the TEM images will include both compositional and strain components but these values are certainly smaller than those deduced from AFM samples [8.1 nm (seed layer) and 11.5 $\mathrm{nm}$ (second layer)] grown under the same conditions [Fig. 2(b)].

With an increase in the size of capped QDs, one would expect an increase in the emission wavelength, especially 


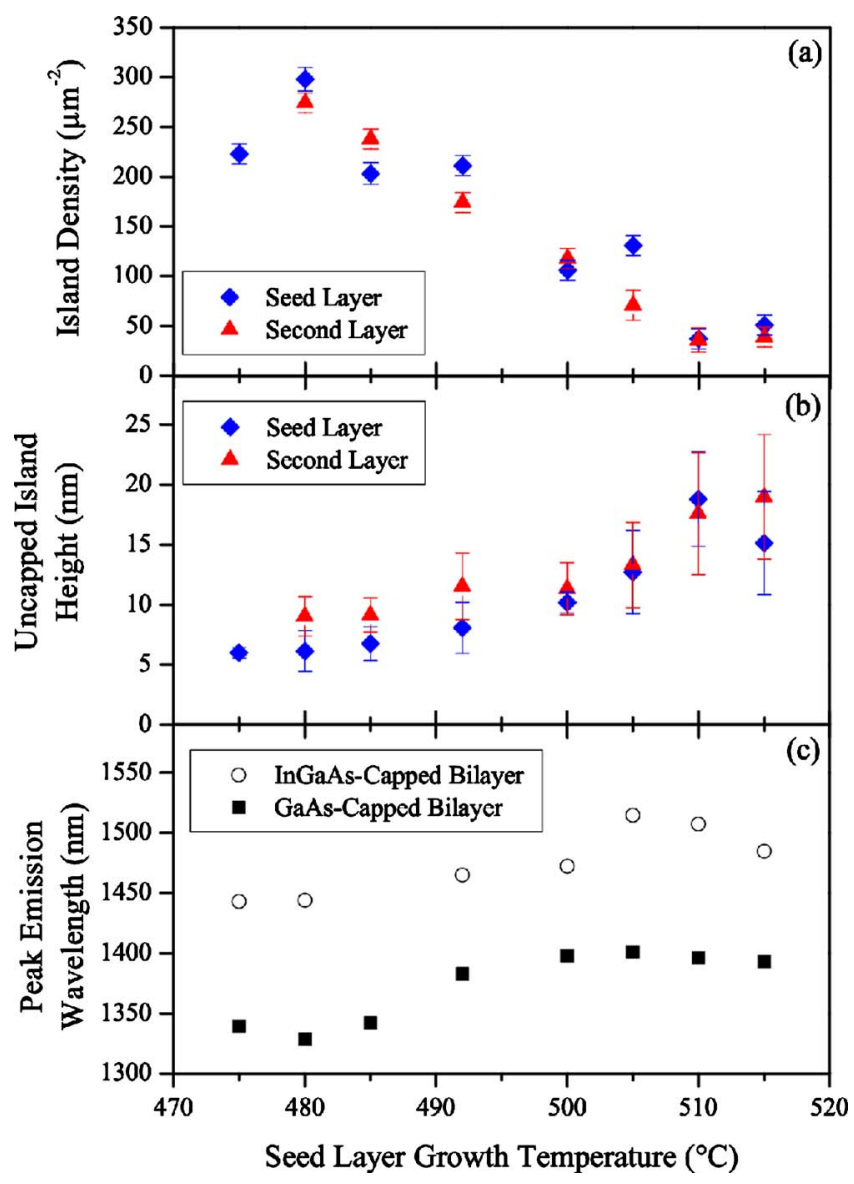

FIG. 2. (Color online) (a) Variation in QD density in the seed layer (diamonds) and the second layer of InAs/GaAs QD bilayers (triangles) with change in the seed layer growth temperature. Note that the growth temperature of the second layer remains the same $\left(467^{\circ} \mathrm{C}\right)$ for all bilayer samples. (b) Variation in average QD height in the seed layer (diamonds) and the second layer of InAs/GaAs QD bilayers (triangles) with change in the seed layer growth temperature. The larger error bars for the lower density samples (high seed layer temperature) are due to the smaller number of QDs analyzed. (c) Variation in peak room temperature emission from InAs/GaAs QD bilayers capped either by GaAs (closed squares) or additionally by an InGaAs strain-reducing layer (open circles) with change in the seed layer growth temperature.

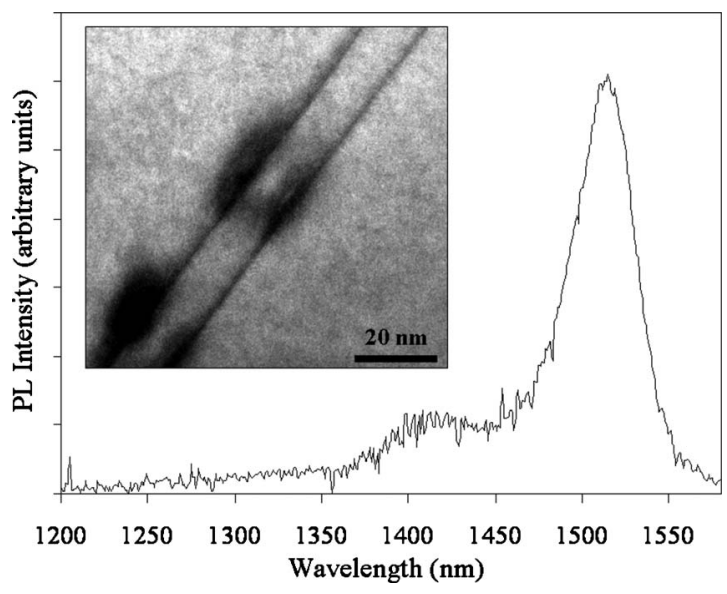

FIG. 3. Room temperature PL spectrum obtained from an InAs/GaAs QD bilayer with the seed layer grown at $505^{\circ} \mathrm{C}$ and the second layer capped by $4 \mathrm{~nm} \mathrm{In}_{0.26} \mathrm{Ga}_{0.74} \mathrm{As}$. Noise around $1350-1400 \mathrm{~nm}$ is due to water absorption. Inset: bright field TEM image of a cross-section of the QD bilayer. since the low growth temperature for the second layer (467 ${ }^{\circ} \mathrm{C}$ for all samples) will suppress $\mathrm{In} / \mathrm{Ga}$ intermixing. Figure 2(c) shows that the peak room temperature PL emission obtained from the GaAs-capped bilayer samples increases from $1340 \mathrm{~nm}$ up to a saturation value of around $1400 \mathrm{~nm}$ with increasing growth temperature of the seed layer. For single layers, such saturation in emission wavelength is due to saturation in QD size ${ }^{17}$ but the AFM data presented in Fig. 2(b) indicates that this is not the case here. We speculate that the saturation in wavelength may occur despite the increase in the size of the QDs if the increased size of the seed layer QDs modifies the strain field experienced by the second layer QDs, leading to increased In/Ga intermixing or strain-related modification of the energy levels in these QDs. Alternatively, the second layer QDs may reach a threshold in size beyond which dislocation occurs, placing a limit on the maximum size of the emissive QDs. The maximum peak wavelength is comparable with the longest reported emission wavelengths for GaAs-capped InAs/ GaAs QDs. ${ }^{25,26}$ However in these previous studies, the QDs were grown at extremely low rates, resulting in an areal density an order of magnitude lower than for the bilayers discussed here. The emission wavelength of these bilayers is still longer than that reported for many InGaAs-capped or dots-in-a-well structures and yet the bilayers do not contain InGaAs strain-reducing layers. When such layers are incorporated, by initially capping the second layer QDs with $4 \mathrm{~nm}$ $\mathrm{In}_{0.26} \mathrm{Ga}_{0.74} \mathrm{As}$, the peak emission of the bilayers is further extended by $\sim 100 \mathrm{~nm}$. Figure 3 shows a room temperature PL spectrum obtained from an InGaAs-capped bilayer QD sample where the seed layer was grown at $505{ }^{\circ} \mathrm{C}$. The peak emission for this sample occurs at $1515 \mathrm{~nm}$, which is the longest wavelength reported for a QD bilayer and is comparable with the longest wavelengths obtained from any QDs grown on GaAs substrates. ${ }^{12-16}$ The emission has a narrow PL linewidth of $22 \mathrm{meV}$, which is consistent with previous reports of narrow emission linewidths from InAs/GaAs bilayers $^{4,27}$ and indicates a high degree of uniformity of the QDs. Emission from the seed layer is suppressed due to efficient electronic coupling between the layers: room temperature emission from the seed layer is expected to be around $1250 \mathrm{~nm}$ for QDs grown under these conditions. The suppression of seed layer emission also persists to low temperatures (not shown). From the TEM image inset to Fig. 3, we note that although the spacer layer between the two QD layers is $10 \mathrm{~nm} \mathrm{GaAs}$, the actual barrier thickness between a pair of vertically correlated QDs is around $5 \mathrm{~nm}$. Previous studies have also demonstrated efficient electronic coupling between QD layers separated by a GaAs spacer layer of 10 $\mathrm{nm}$ (Refs. 2 and 28) and an excitation transfer time of $20 \mathrm{ps}$ has been estimated for bilayer QDs with a similar separation, which is short compared to ground state (GS) radiative lifetime of the seed layer QDs. ${ }^{29}$ Further extension of the emission wavelength may be possible by optimization of the InGaAs cap $^{30}$ but our initial studies indicate that capping with a higher In-content or thicker InGaAs cap results in a significant deterioration in PL intensity. 

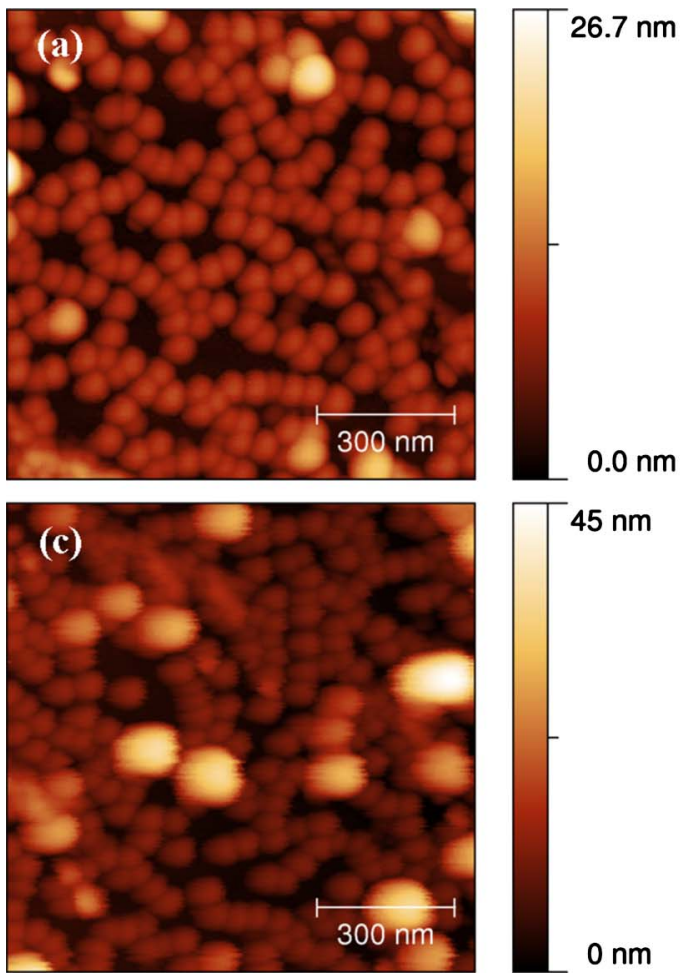
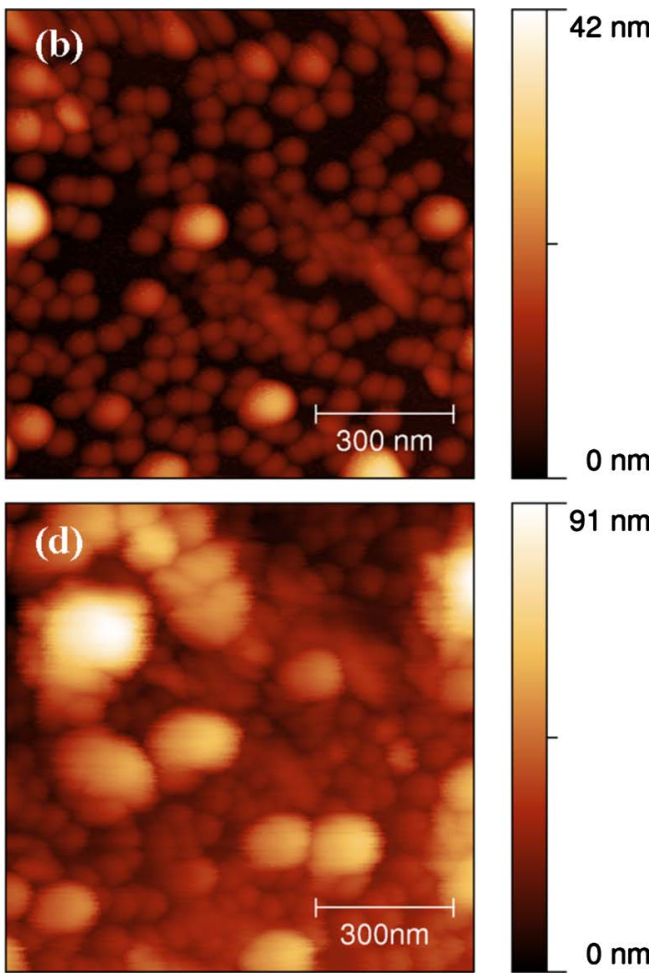

FIG. 4. (Color online) Plan view $1 \times 1 \mu \mathrm{m}^{2} \mathrm{AFM}$ images obtained from InAs/GaAs QD bilayers with seed layer grown at $475{ }^{\circ} \mathrm{C}$ and InAs coverage in the second layer (grown at $467^{\circ} \mathrm{C}$ ) of (a) $3.7 \mathrm{ML}$, (b) $4.5 \mathrm{ML}$, (c) $6.1 \mathrm{ML}$, and (d) $9.0 \mathrm{ML}$.

\section{B. Increasing InAs coverage in the second layer}

Achieving room temperature emission from InAs/GaAs QDs beyond $1500 \mathrm{~nm}$ without recourse to InGaAs metamorphic buffer layers ${ }^{14}$ or complex quaternary strain-reducing layers such as InGaNAs (Ref. 15) or InGaAsSb (Ref. 16) is of interest for device applications, although for many of these applications an increased QD density is also desirable. One would expect that the size of QDs in high density samples could be increased by depositing additional material. However, for single layer growth there is saturation in both QD size and emission wavelength as InAs coverage is increased ${ }^{17}$ and alongside the stable ensemble of coherent islands, a small number of large, irregular InAs islands form. These large islands rapidly grow in size as more material is deposited. Frigeri et al. ${ }^{18}$ have observed the onset of these irregular islands in single layer InAs/GaAs QD growth after 2.4 ML InAs deposition, with the density of these islands rising from $2 \mu \mathrm{m}^{-2}$ for $2.4 \mathrm{ML}$ InAs to $15 \mu \mathrm{m}^{-2}$ for $2.9 \mathrm{ML}$ InAs coverage. A low density $\left(1.2 \mu \mathrm{m}^{-2}\right)$ of large islands can be seen in the AFM image of the seed layer grown at $480{ }^{\circ} \mathrm{C}$ [Fig. 1(a)]. The appearance of increased numbers of these large islands also coincides with the InAs coverage required for significant defect formation in InAs/GaAs QD layers, ${ }^{19}$ and an accompanying decrease in PL intensity from capped samples. ${ }^{18}$ As the InAs coverage is further increased, the irregular islands act as a strain-free sink for surface adatoms during growth, and material migrates away from normal, coherent islands and accumulates almost exclusively in the large islands. ${ }^{20}$ The bilayers discussed in Sec. III A are grown using a higher InAs coverage in the second layer (3.3 $\mathrm{ML}$ ) than is typical for single QD layers but despite this the density of large, irregular islands in the second layer of the bilayers remains an order of magnitude lower than reported for single layers, ${ }^{18}$ around $1-2 \mu \mathrm{m}^{-2}$. We tentatively ascribe this to strain relief provided by the seed layer which inhibits the onset of irregular islands, ${ }^{31}$ although the formation of these islands may also be dependent on the As overpressure during growth.

To investigate the effect of increased InAs coverage in the second layer of the bilayers, samples were grown using a high density seed layer (grown under the same conditions as outlined earlier, with a seed layer growth temperature of $475^{\circ} \mathrm{C}$ ) and with an InAs coverage in the second layer ranging from 3.7-9.0 ML. Figure 4 shows $1 \times 1 \mu \mathrm{m}^{2}$ AFM images obtained from a selection of these samples. Figure 4(a) is from the sample grown with 3.7 ML InAs coverage in the second layer: the majority of the islands are of a similar size and shape, with only a few islands significantly taller than the others, although the number of these large islands is increased from that seen for samples with 3.3 ML InAs coverage. The average height of the regular islands is $8.5 \mathrm{~nm}$, which is comparable with the average island height for reasonably high density bilayers presented in Fig. 2(b). As more InAs is deposited the proportion of regular-sized islands diminishes while the number and size of large, irregular islands increases, at least up to $9.0 \mathrm{ML}$, the largest InAs coverage used in this series of samples. At this InAs coverage, shown in Fig. 4(d), the majority of the deposited material has accumulated in the irregular islands and accounts for roughly half of the sample surface, although some normal-sized islands remain. This is in contrast to previous reports for single layer QD growth, where coverage beyond $\sim 4 \mathrm{ML}$ results only in 


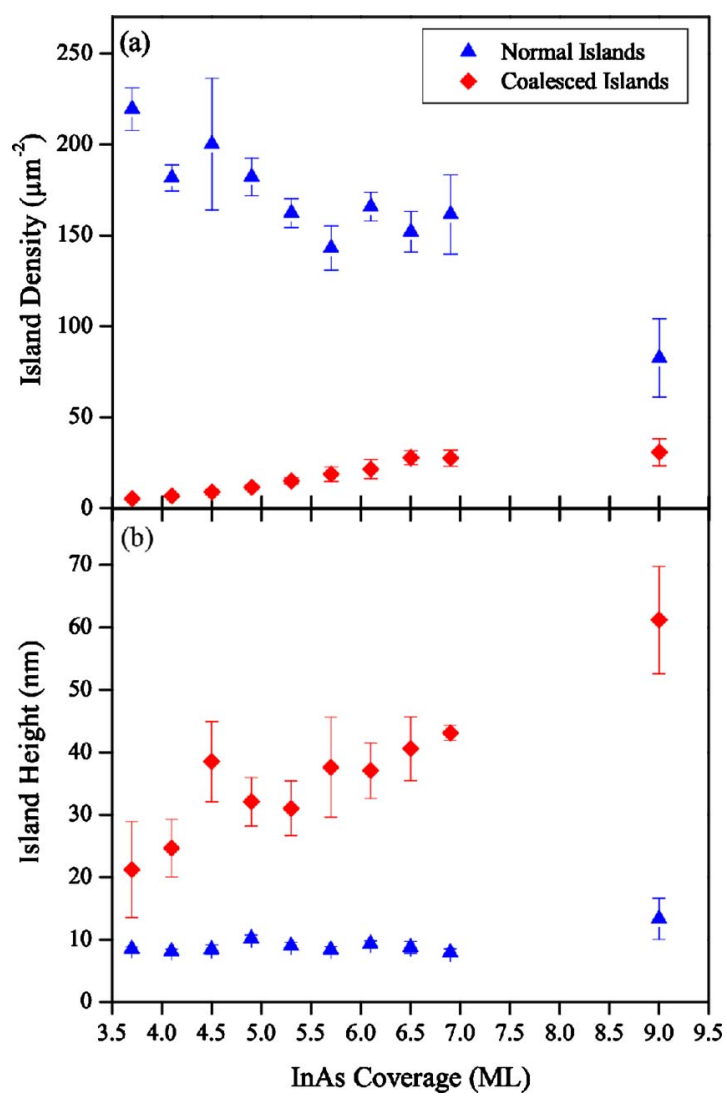

FIG. 5. (Color online) (a) Density and (b) height of regular-sized islands (triangles) and large, coalesced islands (diamonds) in the second layer of QD bilayer as InAs coverage is increased from 3.7 to 9.0 ML.

large, irregular islands. ${ }^{20}$ We conclude that the underlying seed layer provides robust island nucleation sites that can retain adatoms despite the presence of nearby dislocated islands. Previous theoretical analysis of strain in bilayers suggests that underlying QDs can have a significant influence on In diffusion during second layer growth: for the GaAs spacer thickness used here, the In diffusion coefficient is reduced to less than $80 \%$ above a buried QD compared with its value for an unstrained $c(4 \times 4)$ GaAs surface. ${ }^{32}$

Figure 5(a) summarizes the AFM data and shows the density of both the normal and the large, coalesced islands in the second layer of the bilayers as the InAs coverage is increased. The density of normal islands decreases as the number of coalesced islands increases and account for most of the surface area. Figure 5(b) shows the island height for the two populations as the InAs coverage is increased. Although the size of the coalesced islands increases as more material is added, the height of the regular islands remains roughly constant at less than $10 \mathrm{~nm}$. This is further illustrated by PL obtained from capped samples. Figure 6(a) shows that the room temperature peak emission wavelength of the GS from all samples is around $1350 \mathrm{~nm}$ and does not increase as the InAs coverage in the second layer is increased, which is consistent with the constant size of the regular islands in the AFM samples. Surprisingly, the room temperature PL obtained from all samples is of a comparable intensity to conventional (seed layer only) QD samples, even for 9 ML InAs coverage, in marked contrast to single layers where disloca-

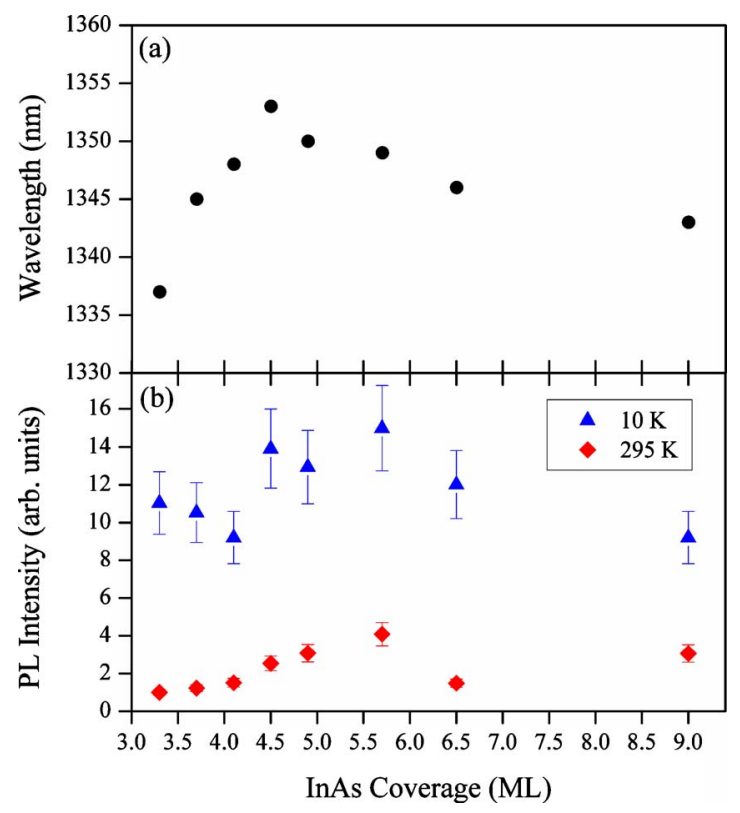

FIG. 6. (Color online) (a) GS PL peak emission wavelength and (b) GS PL peak emission intensity obtained from QD bilayers as InAs coverage in the second layer is increased from 3.3 to $9.0 \mathrm{ML}$.

tion formation leads to a rapid reduction in PL intensity for InAs coverage above 3 ML InAs. ${ }^{18}$ Figure 6(b) shows the peak GS PL intensity obtained from GaAs-capped samples at both low temperature $(10 \mathrm{~K})$ and room temperature $(295 \mathrm{~K})$. The PL intensity at low temperature is approximately constant for all samples while at room temperature the PL intensity is reduced by about a factor of 10 from the low temperature value. A reduction in PL intensity due to the reduction in density of regular QDs with increasing InAs coverage which is seen in the AFM analysis [Fig. 5(a)] is not resolved in our PL measurements, with the variation in PL intensity within that expected for sample-to-sample variations. A similar variation in PL intensity was also observed from nominally identical GaAs/AlGaAs multiple quantum well samples grown for calibration purposes over the same period as these QD samples. We speculate that the reasonably high PL intensity obtained from all samples may be a consequence of efficient carrier capture and transfer from the seed layer to coherent QDs in the second layer providing a source of carriers for radiative recombination.

\section{SUMMARY}

We have investigated two aspects of InAs/GaAs QD bilayer growth conditions and their influence on QD size, with the intention of obtaining larger QDs with long emission wavelengths. This can effectively be achieved by varying the growth conditions for the seed layer, thereby altering the template for second layer growth. Efficient strain coupling ensures that a change in seed layer QD density results in a similar change in the QD density in the upper layer of bilayer structures, and as the QD density is reduced the QDs in the upper layer are increased in size, leading to an increase in the peak emission wavelength to a maximum value of $1400 \mathrm{~nm}$ at room temperature for GaAs-capped bilayers and $1515 \mathrm{~nm}$ for InGaAs capped layers. However, depositing more InAs to 
the second layer of high density QD bilayers does not significantly extend the emission wavelength as the extra material accumulates in coalesced islands but the presence of the seed layer maintains a population of regular, emissive QDs, providing room temperature luminescence even for extremely high InAs coverage in the second layer.

${ }^{1}$ A. E. Zhukov, A. R. Kovsh, N. A. Maleev, S. S. Mikhrin, V. M. Ustinov, A. F. Tsatsul'nikov, M. V. Maximov, B. V. Volovik, D. A. Bedarev, Y. M. Shernyakov, P. S. Kop'ev, Z. I. Alferov, N. N. Ledentsov, and D. Bimberg, Appl. Phys. Lett. 75, 1926 (1999).

${ }^{2}$ E. C. Le Ru, A. J. Bennett, C. Roberts, and R. Murray, J. Appl. Phys. 91 , 1365 (2002)

${ }^{3}$ I. Mukhametzhanov, R. Heitz, J. Zeng, P. Chen, and A. Madhukar, Appl. Phys. Lett. 73, 1841 (1998).

${ }^{4}$ E. C. Le Ru, P. Howe, T. S. Jones, and R. Murray, Phys. Rev. B 67, 165303 (2003).

${ }^{5}$ G. Burkard, G. Seelig, and D. Loss, Phys. Rev. B 62, 2581 (2000).

${ }^{6}$ M. Bayer, P. Hawrylak, K. Hinzer, S. Fafard, M. Korkusinski, Z. R. Wasilewski, O. Stern, and A. Forchel, Science 291, 451 (2001).

${ }^{7}$ Q. Xie, A. Madhukar, P. Chen, and N. P. Kobayashi, Phys. Rev. Lett. 75, 2542 (1995).

${ }^{8}$ L. H. Li, M. Rossetti, A. Fiore, and G. Patriarche, Electron. Lett. 42, 638 (2006).

${ }^{9}$ R. Murray, D. Childs, S. Malik, P. Siverns, C. Roberts, J.-M. Hartmann, and P. Stavrinou, Jpn. J. Appl. Phys., Part 1 38, 528 (1999).

${ }^{10}$ K. Nishi, H. Saito, S. Sugou, and J.-S. Lee, Appl. Phys. Lett. 74, 1111 (1999).

${ }^{11}$ V. M. Ustinov, N. A. Maleev, A. E. Zhukov, A. R. Kovsh, A. Y. Egorov, A. V. Lunev, B. V. Volovik, I. L. Krestnikov, Y. O. Musikhin, N. A. Bert, P. S. Kop'ev, Z. I. Alferov, N. N. Ledentsov, and D. Bimberg, Appl. Phys. Lett. 74, 2815 (1999).

${ }^{12}$ J. Tatebayashi, M. Nishioka, and Y. Arakawa, Appl. Phys. Lett. 78, 3469 (2001).

${ }^{13}$ T.-P. Hsieh, P.-C. Chiu, J.-I. Chyi, N.-T. Yeh, W.-J. Ho, W.-H. Chang, and T.-M. Hsu, Appl. Phys. Lett. 87, 151903 (2005)

${ }^{14}$ N. N. Ledentsov, A. R. Kovsh, A. E. Zhukov, N. A. Maleev, S. S.
Mikhrin, A. P. Vasil'ev, E. S. Semenova, M. V. Maximov, Y. M. Shernyakov, N. V. Kryzhanovskaya, V. M. Ustinov, and D. Bimberg, Electron. Lett. 39, 1126 (2003).

${ }^{15}$ M. Richter, B. Damilano, J.-Y. Duboz, J. Massies, and A. D. Wieck, Appl. Phys. Lett. 88, 231902 (2006).

${ }^{16}$ D. Guimard, S. Tsukamoto, M. Nishioka, and Y. Arakawa, Appl. Phys. Lett. 89, 083116 (2006).

${ }^{17}$ P. B. Joyce, T. J. Krzyzewski, G. R. Bell, T. S. Jones, E. C. Le Ru, and R. Murray, Phys. Rev. B 64, 235317 (2001).

${ }^{18}$ P. Frigeri, L. Nasi, M. Prezioso, L. Seravalli, G. Trevisi, E. Gombia, R. Mosca, F. Germini, C. Bocchi, and S. Franchi, J. Appl. Phys. 102, 083506 (2007).

${ }^{19}$ J. S. Wang, J. F. Chen, J. L. Huang, P. Y. Wang, and X. J. Guo, Appl. Phys. Lett. 77, 3027 (2000).

${ }^{20}$ D. Leonard, K. Pond, and P. M. Petroff, Phys. Rev. B 50, 11687 (1994).

${ }^{21}$ P. Howe, E. C. Le Ru, E. Clarke, R. Murray, and T. S. Jones, J. Appl. Phys. 98, 113511 (2005).

${ }^{22}$ P. B. Joyce, T. J. Krzyzewski, G. R. Bell, B. A. Joyce, and T. S. Jones, Phys. Rev. B 58, R15981 (1998).

${ }^{23}$ P. B. Joyce, T. J. Krzyzewski, G. R. Bell, T. S. Jones, S. Malik, D. Childs, and R. Murray, Phys. Rev. B 62, 10891 (2000).

${ }^{24}$ P. B. Joyce, T. J. Krzyzewski, G. R. Bell, and T. S. Jones, Appl. Phys. Lett. 79, 3615 (2001).

${ }^{25}$ B. Alloing, C. Zinoni, V. Zwiller, L. H. Li, C. Monat, M. Gobet, G. Buchs, A. Fiore, E. Pelucchi, and E. Kapon, Appl. Phys. Lett. 86, 101908 (2005).

${ }^{26}$ D. Guimard, H. Lee, M. Nishioka, and Y. Arakawa, Appl. Phys. Lett. 92, 163101 (2008)

${ }^{27}$ Z. Mi and P. Bhattacharya, J. Appl. Phys. 98, 023510 (2005).

${ }^{28}$ P. Howe, E. C. Le Ru, E. Clarke, B. Abbey, R. Murray, and T. S. Jones, J. Appl. Phys. 95, 2998 (2004).

${ }^{29}$ R. Heitz, I. Mukhametzhanov, P. Chen, and A. Madhukar, Phys. Rev. B 58, R10151 (1998).

${ }^{30}$ L. Seravalli, M. Minelli, P. Frigeri, S. Franchi, G. Guizzetti, M. Patrini, T. Ciabattoni, and M. Geddo, J. Appl. Phys. 101, 024313 (2007).

${ }^{31}$ I. Daruka and A.-L. Barabási, Phys. Rev. Lett. 79, 3708 (1997).

${ }^{32}$ S. Tomić, P. Howe, N. M. Harrison, and T. S. Jones, J. Appl. Phys. 99, 093522 (2006) 